Published in: International Journal of Bifurcation and Chaos, 26 (2), Paper 1650023. 11 p. (2016) International Journal of Bifurcation and Chaos

(c) World Scientific Publishing Company

\title{
MULTI-BAKER MAP AS A MODEL OF DIGITAL PD CONTROL
}

\author{
GÁBOR CSERNÁK \\ MTA-BME Research Group on Dynamics of Machines and Vehicles, Müegyetem rkp. 5. \\ H-1521 Budapest, Hungary \\ csernak@mm.bme.hu \\ GERGELY GYEBRÓSZKI \\ Department of Applied Mechanics, \\ Budapest University of Technology and Economics, Müegyetem rkp. 5. \\ H-1521 Budapest, Hungary \\ gyebro@mm.bme.hu \\ GÁBOR STÉPÁN \\ Department of Applied Mechanics, \\ Budapest University of Technology and Economics, Müegyetem rkp. 5. \\ H-1521 Budapest, Hungary \\ stepan@mm.bme.hu \\ Received
}

\begin{abstract}
Digital stabilization of unstable equilibria of linear systems may lead to small amplitude stochastic-like oscillations. We show that these vibrations can be related to a deterministic chaotic dynamics induced by sampling and quantization. A detailed analytical proof of chaos is presented for the case of a PD controlled oscillator: it is shown that there exists a finite attracting domain in the phase-space, the largest Lyapunov exponent is positive and the existence of a Smale horseshoe is also pointed out. The corresponding two-dimensional micro-chaos map is a multi-baker map, i.e., it consists of a finite series of baker's maps.
\end{abstract}

Keywords: Digital control, hybrid system, chaos proof, Smale horseshoe, micro-chaos

\section{Introduction}

Digital control systems often exhibit strange, stochastic-like behaviour [Kuo, 1977; Chen \& Dong, 1998; Delchamps, 1990]. The occuring small amplitude vibrations are usually considered as noise in practice [Widrow \& Kollár, 2008], however, their source may be deterministic chaotic dynamics. Due to the digital effects, i.e., the sampling and the round-off (or quantization), the behavior of full-state feedback controlled linear systems can be described by piecewise linear maps, so-called micro-chaos maps. It is easy to show in the general case that there exists a finite size absorbing sphere - global attractor - in the phase-space of these maps. Moreover, if an unstable equilibrium is to be stabilized by the control, the largest Lyapunov exponent is positive. However, the proof of topological transitivity and the existence of an infinity of unstable periodic orbits was rather difficult in the special cases, considered so far. It was proved in the 
mid-nineties [Haller \& Stépán, 1996] that the differential (D) control of the motion of a block sliding on a surface characterized by Stribeck friction leads to small amplitude chaotic vibrations. The motion of the block was described by a one-dimensional micro-chaos map. By adding dry friction to the system, the permanently chaotic vibrations turn to transient chaotic ones. We developed a method for the exact calculation of the mean lifetime of chaotic transients in this case [Csernák \& Stépán, 2005]. If one takes into account a processing delay that is just equal to the sampling period, the micro-chaos map becomes two-dimensional, but the attractors become one-dimensional. The proof of chaos was presented for this map and the lifetime estimation algorithm was generalized in [Csernák \& Stépán, 2010] and [Csernák \& Stépán, 2007]. A similar 2D map was introduced in [Garay et al., 2008] as a model of hysteresis. It was shown that the considered 2D map can be transformed to a 1D map. This property was exploited for the determination of various properties of the system.

In the present paper, a detailed proof of chaos is given for the case of a PD controlled linear oscillator. The corresponding two-dimensional micro-chaos map is a multi-baker map, i.e., it consists of a finite series of baker's maps. As a consequence of this property, several disconnected strange attractors may coexist far from the desired state of the system. Besides the proof of the existence of an attractor and the sensitive dependence on initial conditions, the existence of a Smale horseshoe is also pointed out analytically.

We believe that certain concepts, presented in this paper, can be extended to other areas of science. For example, similar hybrid models were introduced in population dynamics [Milton \& Bélair, 1990] and neurology [Cabrera \& Milton, 2004].

\section{Mathematical Model}

Consider a linear oscillator with negative stiffness, e.g. an inverted pendulum, whose equation of motion is linearized about the unstable equilibrium. The equation of motion of the controlled system assumes the following form:

$$
M \ddot{x}(t)+c \dot{x}(t)-k x(t)=u,
$$

where $x$ is the general coordinate, $M$ denotes (generalized) mass, $c \geq 0$ is the damping coefficient, $k>0$ is the (generalized) stiffness, while $u$ denotes the control force. PD control is applied on the system - i.e., a virtual spring and a virtual dashpot - in order to stabilize the upright position $(x=0, \dot{x}=0)$, according to the output of a digital control system.

\subsection{Effect of Sampling}

During sampling, the position and velocity are measured periodically, with period $\tau$. The sampled values are used to calculate the control force. We assume that the control force $u$ is kept constant between two successive sampling instants $t_{j} \equiv j \tau$ and $t_{j+1} \equiv(j+1) \tau$, see Fig. 1. Although a certain amount of time is necessary for the calculation of the control force, we assume that this processing delay is negligible.

Thus, if quantization is not considered, the equation of motion of the controlled system becomes piecewise linear on the intervals $t \in[j \tau,(j+1) \tau)$ :

$$
\ddot{x}(t)+\frac{c}{M} \dot{x}(t)-\frac{k}{M} x(t)=-\frac{\tilde{P} x_{j}+\tilde{D} \dot{x}_{j}}{M},
$$

where $\dot{x}_{j}=\dot{x}(j \tau), j \in \mathbb{N}$, while $\tilde{P}$ and $\tilde{D}$ denote the proportional and differential gains, respectively. Thus, the control force can be given as $u_{j}=-\left(\tilde{P} x_{j}+\tilde{D} \dot{x}_{j}\right), t \in[j \tau,(j+1) \tau)$.

Let us introduce a dimensionless displacement coordinate $y=x / \tilde{h}$, where $\tilde{h}$ is a reference distance - to be defined later -, and a non-dimensional time $T=t / \tau$, where $\tau$ is the sampling time and $t$ is the physical time. With this new time variable, $\dot{\square} \equiv \frac{d}{d t} \square=\frac{1}{\tau} \frac{d}{d T} \square \equiv \frac{1}{\tau} \square^{\prime}$, thus, denoting the differentiation with respect to $T$ by prime, one obtains the following equation of motion for $T \in[j,(j+1))$ :

$$
\frac{1}{\tau^{2}} \tilde{h} y^{\prime \prime}(T)+\frac{c \tilde{h}}{M \tau} y^{\prime}(T)-\frac{k \tilde{h}}{M} y(T)=-\frac{\tilde{P} \tilde{h} \tau y_{j}+\tilde{D} \tilde{h} y_{j}^{\prime}}{M \tau} .
$$




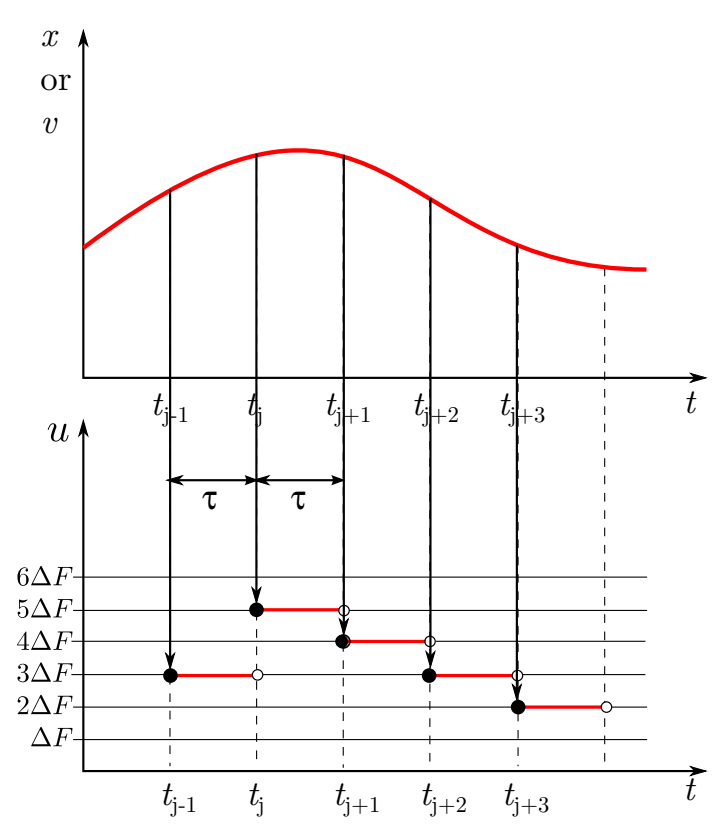

Fig. 1. Sample and hold control scheme with negligible processing delay and quantized control force $u$.

After rearranging terms, the equation assumes the following dimensionless form:

$$
y^{\prime \prime}+2 \beta y^{\prime}-\alpha^{2} y=-\left(P y_{j}+D y_{j}^{\prime}\right),
$$

where

$$
\beta=\frac{c \tau}{2 M}, \quad \alpha=\tau \sqrt{\frac{k}{M}}, \quad P=\frac{\tilde{P} \tau^{2}}{M}, \quad D=\frac{\tilde{D} \tau}{M} .
$$

As it can be seen, the reference distance $\tilde{h}$ does not influence the form of the equation of motion and the dimensionless variables. It is worth noting that parameter $\alpha$ is the ratio of the sampling time and the characteristic time constant of the oscillator. Introducing the notation $\gamma=\sqrt{\alpha^{2}+\beta^{2}}$, the general solution of Eq. (4) assumes the following form in $T \in[j,(j+1))$ :

$$
y(T)=C_{1} e^{(\gamma-\beta) T}+C_{2} e^{(-\gamma-\beta) T}+\frac{P y_{j}+D y_{j}^{\prime}}{\alpha^{2}} .
$$

According to the matching conditions $y(j)=y_{j}$ and $y^{\prime}(j)=y_{j}^{\prime}$, the coefficients - and consequently, $y_{j+1}$ and $y_{j+1}^{\prime}-$ can be expressed by $y_{j}$ and $y_{j}^{\prime}$. Thus, one can construct a linear map

$$
\mathbf{y}_{j+1}=\mathbf{S y}_{j},
$$

where

$$
\mathbf{S}=\frac{1}{e \gamma}\left[\begin{array}{cc}
\frac{\left(\alpha^{2}-P\right)(\beta s+\gamma c)+P e \gamma}{\left(\alpha^{2}-P\right) s} & \frac{\alpha^{2} s-(\beta s+\gamma c-e \gamma) D}{\alpha^{2}}+\left(\beta^{2}+D\right) s
\end{array}\right],
$$

while $\mathbf{y}_{j}=\operatorname{col}\left[y_{j} \quad y_{j}^{\prime}\right], s \equiv \sinh (\gamma), c \equiv \cosh (\gamma)$, and $e \equiv \exp (\beta)$.

For the asymptotic stability of the origin, the roots of the characteristic equation

$$
\mu^{2}+\frac{(s D-2 \gamma c) \alpha^{2}-P(\gamma(e-c)-\beta s)}{e \gamma \alpha^{2}} \mu+\frac{\gamma \alpha^{2}-D e s \alpha^{2}-P(e \beta s-e \gamma c+\gamma)}{e^{2} \gamma \alpha^{2}}=0
$$

have to reside inside the unit circle on the complex plane. To check this condition using the Routh-Hurwicz criterions, we applied a Möbius transformation, i.e., introduced a new variable $\eta$ as $\mu=(\eta+1) /(\eta-1)$. After substitution, we obtained another characteristic equation $(\operatorname{Re}(\eta)<0 \Longleftrightarrow|\mu|<1)$ in the form

$$
b_{2} \eta^{2}+b_{1} \eta+b_{0}=0
$$


where

$$
\begin{aligned}
& b_{2}=\left(P-\alpha^{2}\right) \gamma\left(2 c e-1-e^{2}\right), \\
& b_{1}=-\alpha^{2}\left(-2 s D e+2 \gamma\left(1-e^{2}\right)\right)-2 P(e c \gamma-e s \beta-\gamma), \\
& b_{0}=\alpha^{2}\left(\gamma\left(e^{2}+1+2 c e\right)-2 s D e\right)-P\left(2 e s \beta+\gamma\left(1-e^{2}\right)\right) .
\end{aligned}
$$

The origin is stable if these coefficients are greater than zero. Note, that since $\exp (\beta)>0$ and $\gamma>0$, $2 c e-1-e^{2}>0$ is fulfilled and the condition $b_{2}>0$ corresponds to $P>\alpha^{2}$ independently of the damping - as in [Csernák \& Stépán, 2011], where the undamped case was examined.

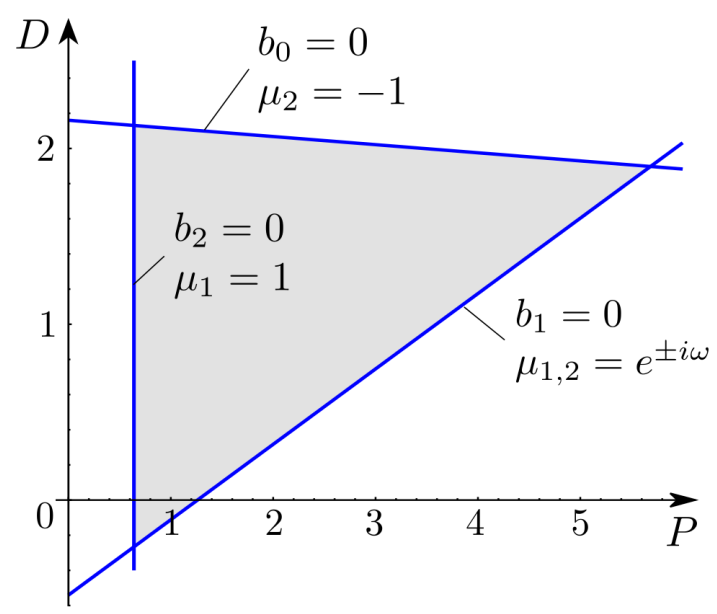

Fig. 2. The domain of asymptotic stability of the system without processing delay and quantization (shaded) at $\alpha=0.8$ and $\beta=0.3$.

The domain of stability is shown in Fig. 2 for $\alpha=0.8$ and $\beta=0.3$. If the stable domain disappears during the continuous variation of parameters, the three border lines must have a common intersection point. This situation would arise only at $\beta \rightarrow-\infty$. According to the formulae, the domain of stability would disappear at $\alpha=0$, too. However, solution (6) and the stability calculation are not valid if $\alpha=0$. For the treatment of this case, see [Csernák \& Stépán, 2010] and [Csernák \& Stépán, 2012]. Thus, if parameter $\alpha$ is strictly positive and $\beta$ is finite, the equilibrium can be asymptotically stabilized at every positive value of the sampling time $\tau$ - with properly chosen gains $P$ and $D$. It is worth noting here that the equation of motion of a real inverted pendulum is nonlinear. Thus, if one wants to keep the pendulum in the vicinity of the upright position in order to apply the linearized equations, parameter $\alpha$ - and consequently, the sampling time $\tau$ - must be rather small. At $b_{2}=0$ one of the characteristic multipliers becomes 1 (saddle node bifurcation in the nonlinear system). At $b_{0}=0$ one of them becomes -1 (period doubling), while at $b_{1}=0$ the characteristic roots become complex with unit modulus (Neimark-Sacker bifurcation).

We focus on those parameters at which the eigenvalues of $\mathbf{S}$ are less than one in modulus. In this case the origin of the sampled system is asymptotically stable if the round-off is not taken into account.

\subsection{Quantization - Round-off at the Output}

In a real control system, the signals of the measured quantities $y$ and $y^{\prime}$ are sent to an analog-digital (AD) converter before being processed by the computer. Due to the finite resolution of the AD converter, some information is lost about the state of the system. Similarly, also the output of the control system can assume only discrete values.

Both aforementioned quantization types can be significant depending on the implementation of the control system [Csernák \& Stépán, 2013]. In the present paper, we neglect the effects of rounding during the measurement of position and velocity compared to the rounding of the output. This assumption corresponds to control systems with Pulse Width Modulation (PWM)-driven actuator and analog distance measurement. For example an Arduino Mega microcontroller has 8-bit PWM resolution while 12-bit analog-to-digital resolution is achievable by oversampling. 
In this case the rounded control force must be an integer multiple of a certain resolution $\Delta F$ (cf. Fig. 1):

$$
\ddot{x}(t)+\frac{c}{M} \dot{x}(t)-\frac{k}{M} x(t)=-\frac{\Delta F}{M} \operatorname{Int}\left(\frac{\tilde{P} x_{j}+\tilde{D} \dot{x}_{j}}{\Delta F}\right), t \in[j \tau,(j+1) \tau) .
$$

The odd function Int () rounds towards the origin. Introducing the new dimensionless variables $T=t / \tau$ and $y=M /\left(\Delta F \tau^{2}\right) x$ (i.e., the appropriate choice of the reference distance is $\tilde{h}=\Delta F \tau^{2} / M$, cf. Eq. (3)), one arrives at the equation

$$
y^{\prime \prime}(T)+2 \beta y^{\prime}(T)-\alpha^{2} y(T)=-\operatorname{Int}\left(P y_{j}+D y_{j}^{\prime}\right),
$$

where $T \in[j,(j+1))$ and the parameters are the same as in (5). Thus, only the integer part of the previously calculated contol force is taken into account. Note, that the variation of $\Delta F$ leads merely to the scaling of the phase-space. Consequently, the solutions can be given by the following mapping:

$$
f\left(\mathbf{y}_{\mathbf{i}}\right) \equiv \mathbf{y}_{\mathbf{j}+\mathbf{1}}=\mathbf{U}_{\mathbf{j}}+\mathbf{b} \operatorname{Int}\left(P y_{j}+D y_{j}^{\prime}\right) \equiv \mathbf{U}_{\mathbf{j}}+\mathbf{b} m,
$$

where $m=\operatorname{Int}\left(P y_{j}+D y_{j}^{\prime}\right)$ and $\mathbf{y}_{\mathbf{j}}=\operatorname{col}\left[y_{j} y_{j}^{\prime}\right]$. Matrix $\mathbf{U}$ is the coefficient matrix of the uncontrolled system with unstable origin:

$$
\mathbf{U}=\frac{1}{e \gamma}\left[\begin{array}{cc}
\gamma c+\beta s & s \\
\alpha^{2} s & \gamma c-\beta s
\end{array}\right]
$$

while vector $\mathbf{b}$ is given as

$$
\mathbf{b}=\left[\begin{array}{c}
\frac{e-c}{e \alpha^{2}}-\frac{\beta s}{e \gamma \alpha^{2}} \\
-\frac{s}{e \gamma}
\end{array}\right]
$$

such that $\mathbf{S}=\mathbf{U}+\mathbf{b} \circ\left[\begin{array}{ll}P & D\end{array}\right]^{T}$. Here o denotes the dyadic (Kronecker) product.

The local stability properties of the mapping are determined by the matrix $\mathbf{U}$ with eigenvalues $\lambda_{1,2}^{U}=$ $\exp (-\beta \pm \gamma)$. Since $\gamma>\beta$, the origin is locally unstable and all the existing fixed points are saddle points. The eigenvectors - showing the directions of the stable and unstable manifolds of fixed points, respectively - are

$$
\mathbf{e}_{\mathrm{s}}=\left[\begin{array}{c}
1 \\
-\gamma-\beta
\end{array}\right] \quad \text { and } \quad \mathbf{e}_{\mathrm{u}}=\left[\begin{array}{c}
1 \\
\gamma-\beta
\end{array}\right] .
$$

The evolution of the state of the system can be described by another map, as well:

$$
\mathbf{y}_{\mathbf{j}+\mathbf{1}}=\mathbf{S y}_{\mathbf{j}}-\mathbf{b} \chi_{j},
$$

where $\mathbf{S}$ is given by (8) and $-1<\chi_{j}<1$ is the fractional part of the control force. If the system's state is far from the origin, the effect of round-off is negligible. Thus, the origin is practically stable [Lakshmikantham et al., 1990], since matrix $\mathbf{S}$ governs the system's large-scale behaviour.

As it will be shown in Section 4, the local instability of fixed points and the practical stability of the origin implies that the coefficients $\chi_{j}$ vary irregularly during the evolution of map (17) leading to chaotic vibrations. This argumentation can be extended to more refined control systems, as well: all the fixed points and periodic orbits must be locally unstable in the domains of constant control effort, even if the desired state is practically stable.

\section{Topology of the Phase-space}

The domain of definition of (13) can be divided into discrete bands, according to the value of the number $m$ (see Eq. (13)). The computer sends out the same output signal within such a band, due to the round-off. The bands are separated by the lines

$$
S W_{m}: \quad y^{\prime}=\frac{m-P y}{D}, \quad|m|=1,2,3, \ldots,
$$


thus, these switching lines cross the $y^{\prime}=0$ line at $y=m / P$.

Figure 3 shows two such switching lines that separate bands $m=4, m=5$ and $m=6$. Two numerically generated strange attractors are also presented close to these lines, that were obtained using different initial conditions.

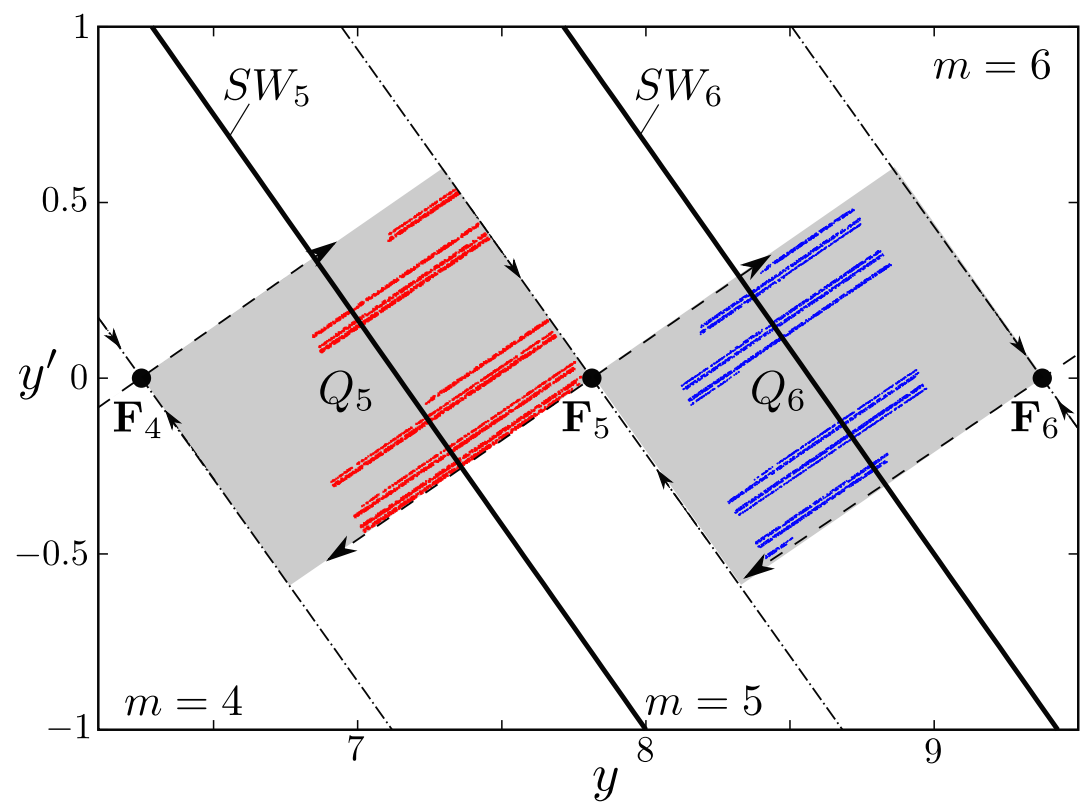

Fig. 3. Two numerically obtained disconnected attractors at the switching lines $S W_{5}$ and $S W_{6}$ (thick solid lines) and the manifolds of the neighbouring fixed points (dashed thin lines) at $\alpha=0.8, \beta=0.3, P=0.7$, and $D=0.6$. The attractors can be encapsulated in parallelograms $Q_{5}$ and $Q_{6}$, defined by the manifolds.

Due to the dissipative nature of the map ( $\operatorname{since} \beta>0$ ), the phase-space volumes are contracting, tending to a structure of zero measure. Since the unstable manifolds of the fixed points lead to the attractors, each chaotic attractor can be considered as the closure of one of the branches of the unstable manifolds. Thus, the attractor becomes the direct product of threads along the unstable manifold and a Cantor set-like structure along the stable manifold [Farmer et al., 1983]. Consequently, the fractal dimension of the attractors must be between 1 and 2 (see Section 4.2).

The disconnected attractors - that are nested by the stable and unstable manifolds of the neighbouring fixed points - are found at the crossings of the switching lines and the $y^{\prime}=0$ line, i.e., at $\left(y_{m} y_{m}^{\prime}\right)^{T}=$ $(m / P 0)^{T}$. Thus, these attractors can also be indexed by the corresponding integer $m$.

The fixed points of map (13) can be given by straightforward calculation:

$$
\mathbf{F}_{m} \equiv(\mathbf{I}-\mathbf{U})^{-1} \mathbf{b} m=\left[\frac{m}{\alpha^{2}} 0\right]^{T}
$$

where $\mathbf{I}$ denotes the unit matrix. Note, that the velocity $y^{\prime}$ is necessarily zero at the fixed points. The fixed point $\mathbf{F}_{m}$ must be in band $m$, thus

$$
\frac{|m|}{P} \leq \frac{|m|}{\alpha^{2}}<\frac{|m|+1}{P}
$$

must be fulfilled to have a real fixed point in the $m$ th band. If the fixed point $\mathbf{F}_{m}$ crosses the switching line at $|m| / \alpha^{2}=(|m|+1) / P$, a border collision bifurcation occurs and the fixed point becomes virtual. It can be seen that the dimensionless parameters (5) depend on the sampling time $\tau$ such that its variation does not change the structure of fixed points. However, the increase of parameter $\tau$ means that the average distance of subsequent points during the iteration of the micro-chaos map also increases. Thus, the size of the strange attractors increases as well, which may lead to the merging of these structures. 
For the detailed examination of the topology of the phase-space, we searched for Smale horseshoe structures. As initial domains, we chose the parallelograms $Q_{r}, r \in \mathbb{N} \backslash\{0\}$ defined by the stable and unstable manifolds $W_{L}^{S}, W_{L}^{U}, W_{R}^{S}$ and $W_{R}^{U}$ of neighbouring fixed points $F_{l}$ and $F_{r}$ (cf. Fig. 3). Here $r$ denotes the index of the switching line $S W_{r}$ between the two fixed points. $l$ and $r$ are the integer numbers corresponding to the integer part of the control force in the bands next to the switching line $S W_{r}$. Each parallelogram is divided into two trapezoids (denoted by $R$ on the right and $L$ on the left, see the crosshatched regions in Fig. 4) by a switching line. The vertices of the trapezoid $L$ are the fixed point $F_{l}$, the intersection point $P^{R U L S}$ of the manifolds $W_{R}^{U}$ and $W_{L}^{S}$, the intersection point $P^{R U S W}$ of $W_{R}^{U}$ and the switching line, and the crossing point $P^{L U S W}$ of manifold $W_{L}^{U}$ and the switching line, see Fig. 4 . The vertices of the right trapezoid can be given similarly by straightforward analytical calculation. The images of trapezoids $L$ and $R$ can be calculated by restricting the dynamics to the corresponding band, i.e., to the case $m=l$ or $m=r$. Thus, we can introduce the restricted versions of the micro-chaos map: $f_{l}=\left.f\right|_{m=l}$ and $f_{r}=\left.f\right|_{m=r}$. The images of the quadrangles $f_{r}(R)$ and $f_{l}(L)$ are stretched along the unstable manifolds, while the pre-images $f_{r}^{-1}(R), f_{l}^{-1}(L), f_{l}^{-1}(R)$ and $f_{r}^{-1}(L)$ are stretched along the stable manifolds. One must be careful during the determination of pre-images, since e.g. $f_{r}^{-1}(L)=\tilde{f}_{r}^{-1}(L) \cap R$, where

$$
\tilde{f}_{r}^{-1}(L)=\left\{\tilde{\mathbf{y}} \mid \tilde{\mathbf{y}}=\mathbf{U}^{-1}(\mathbf{y}-\mathbf{b} r), \mathbf{y} \in L\right\} .
$$

Thus, it may happen that some parts of the calculated pre-image set $\tilde{f}_{r}^{-1}(L)$ are cut away by the switching line $S W_{r}$.

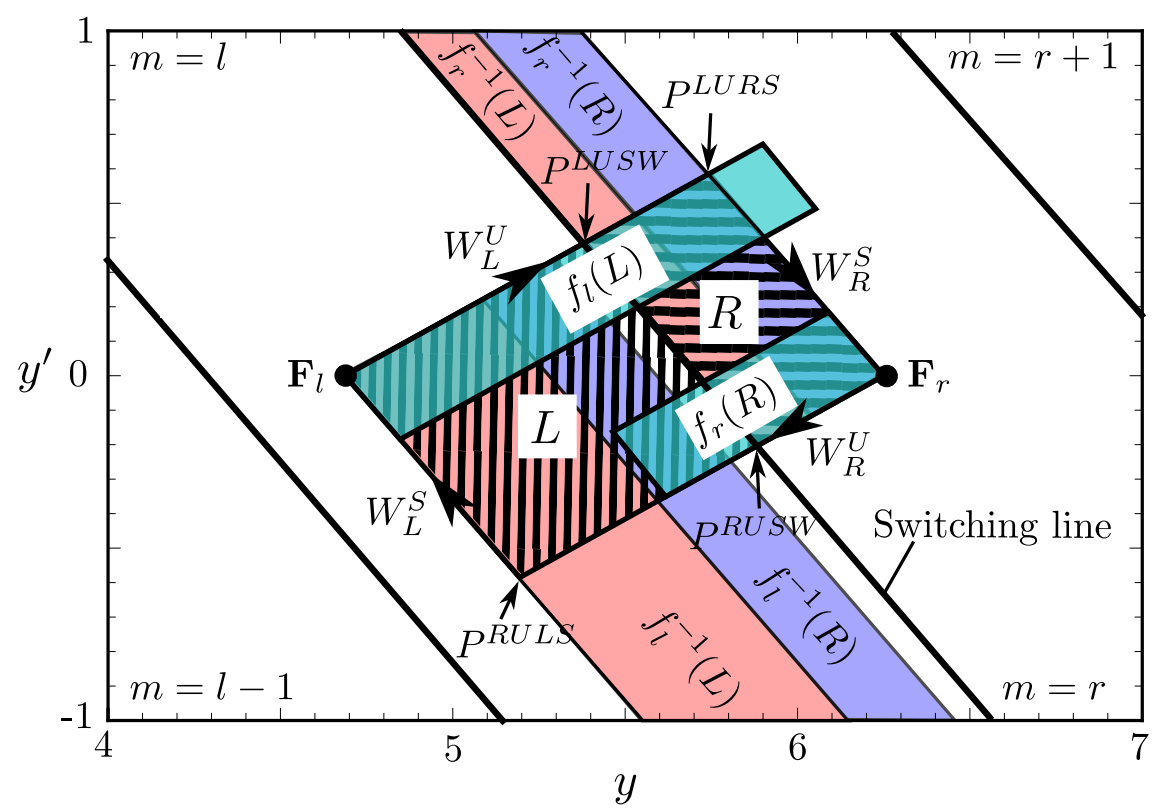

Fig. 4. The schematic picture of the horseshoe-structure in the parallelogram $Q_{r}=L \cup R$. The figure was generated at parameters $r=4, \alpha=0.8, \beta=0.3, P=0.7$ and $D=0.6$.

It is easy to see in Fig. 4 that $f_{l}(L) \backslash Q_{r} \neq \emptyset$, i.e., $f_{l}(L)$ is stretched out from the initial parallelogram. Thus, a chaotic repeller with transient chaotic motion exists in this domain. Several different attractors and repellers may coexist at certain parameters. For instance, Fig. 5 shows four strange objects next to each other. Two of them (at $m=4$ and $m=7$ ) are repellers, while the images of parallelograms do not stretch out from the initial domain at $m=5,6$, thus, two separated attractors exist here - these were already shown in Fig. 3.

\section{Proof of Chaos}

The attractors shown in Fig. 3 are apparently strange, but we cannot state that the solutions are chaotic until this property is mathematically proved. First we will prove that the solutions must stay in the vicinity 


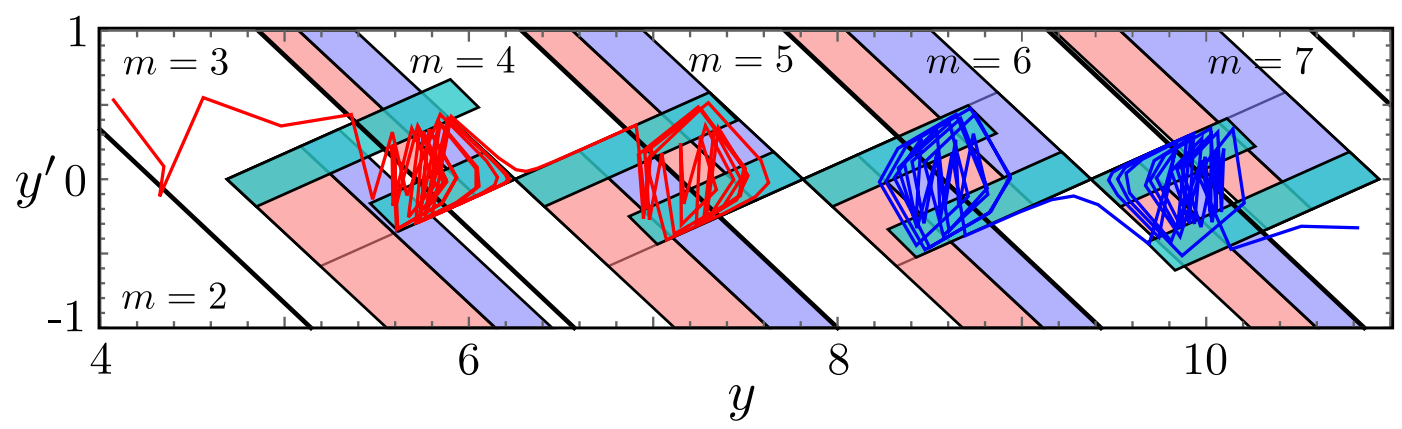

Fig. 5. The horseshoe-structures at the switching lines $m=4,5,6,7$ at $\alpha=0.8, \beta=0.3, P=0.7$ and $D=0.6$. Two trajectories are also shown, leading to the disconnected attractors whose points were presented in Fig. 3.

of the origin, i.e., there is an absorbing sphere which attracts all the trajectories that are far from the origin. This property is important from practical point of view, since the size of the absorbing sphere provides an estimation of the maximal possible control error.

We adopt the following definition of chaos: the dynamical system (13) is chaotic if the solutions of the map exhibit sensitive dependence on initial conditions, the map is topologically transitive (mixing), and the periodic points are dense in a properly chosen set. These conditions are checked in the present section.

\subsection{Existence of an Absorbing Sphere}

Since the origin is practically stable if the gains are chosen from the domain of stability, there must exist a so-called absorbing sphere - a finite attracting domain in the neighbourhood of the origin. In the present section the size of this domain is estimated.

The micro-chaos map (17) can be rewritten to the following form:

$$
\mathbf{y}_{j+1}=\mathbf{S}^{j} \mathbf{y}_{0}-\sum_{k=0}^{j-1} \mathbf{S}^{k} \mathbf{b} \chi_{k} .
$$

Unfortunately, matrix $\mathbf{S}$ is typically a so-called non-normal matrix, i.e., its Euclidean norm (the greatest singular value) can be larger than 1 . Still - since $\mathbf{S}$ is chosen such that its eigenvalues are inside the unit circle - ,

$$
\lim _{j \rightarrow \infty}\left\|\mathbf{S}^{j}\right\|=0
$$

is fulfilled with any kind of norm [Csernák \& Stépán, 2010]. It means that the information about the initial state vanishes and all solutions tend towards the absorbing sphere.

Since the numbers $\chi_{k}$ vary during the application of the micro-chaos map, the series (22) is divergent for almost all initial conditions. The only exceptions are the fixed points of the map. However, one can define a sequence $\tilde{\chi} \equiv \tilde{\chi}_{0}, \tilde{\chi}_{1}, \ldots$ such that (22) becomes convergent and tends to the point $\mathbf{y}_{\infty}$ of the attractor which is farthest from the origin in a chosen metric:

$$
\left\|\mathbf{y}_{\infty}\right\| \equiv \max _{\tilde{\chi}}\left\|-\lim _{j \rightarrow \infty} \sum_{k=0}^{j} \mathbf{S}^{k} \mathbf{b} \tilde{\chi}_{k} .\right\|
$$

If we can give a finite upper estimate for the norm of $\mathbf{y}_{\infty}$, the existence of the finite absorbing sphere is proven. The details of the application of this concept depend on the properties of matrix $\mathbf{S}$ and vector $\mathbf{b}$.

(a) In most of the cases, $\mathbf{S}$ can be expressed as a diagonal matrix $\hat{\mathbf{S}}$ in the basis of eigenvectors. Let $\mathbf{T}$ denote the (in general complex-valued) matrix that is composed of the eigenvectors of $\mathbf{S}$. With this notation, one obtains:

$$
\mathbf{T}^{-1} \mathbf{y}_{\infty}=\sum_{k=0}^{\infty} \mathbf{T}^{-1} \mathbf{S}^{k} \mathbf{T} \mathbf{T}^{-1} \mathbf{b} \tilde{\chi}_{k}
$$


In the basis of the eigenvectors of $\mathbf{S}$,

$$
\mathbf{T}^{-1} \mathbf{b}=\left[\begin{array}{ll}
\tilde{b}_{1} & \tilde{b}_{2}
\end{array}\right]^{T}
$$

Thus,

$$
\mathbf{T}^{-1} \mathbf{y}_{\infty}=\left[\begin{array}{ll}
\sum_{k=0}^{\infty} \lambda_{1}^{k} \tilde{b}_{1} \tilde{\chi}_{k} \\
\sum_{k=0}^{\infty} \lambda_{2}^{k} \tilde{b}_{2} \tilde{\chi}_{k}
\end{array}\right] .
$$

If the eigenvalues $\lambda_{1,2}$ are complex or negative real numbers, the choice $\tilde{\chi}_{k}=1, \forall k$ does not lead to the maximal norm of $\mathbf{y}_{\infty}$. This problem can be overcome if the norms of the eigenvalues and the elements of $\mathbf{T}^{-1} \mathbf{b}$ and $\mathbf{T}$ are considered:

$$
\left\|\mathbf{y}_{\infty}\right\|<\sum_{i=1}^{2}\left\|\sum_{j=1}^{2}\right\| T_{i j}\left\|\frac{\left\|\tilde{b}_{j}\right\|}{1-\left\|\lambda_{j}\right\|}\right\| .
$$

(b) In the rare case, when $\mathbf{S}$ is not diagonalizable, further results can be used from the theory of nonnormal matrices [Trefethen \& Embree, 2005]. Unfortunately the upper estimates that are based on the general theory usually provide unrealistically large numbers. These large numbers cannot be used for the prediction of the behaviour of the real control system.

In summary, $\left\|\mathbf{y}_{\infty}\right\|$ is necessarily finite if the eigenvalues of $\mathbf{S}$ are inside the unit circle, thus, there exists an absorbing sphere.

\subsection{Sensitivity to Initial Conditions}

The eigenvalues of $\mathbf{U}$ are $\lambda_{1,2}^{U}=\exp (-\beta \pm \gamma)$. Since $\gamma>\beta$, the origin is locally unstable and all the existing fixed points are saddle points. Consequently, $\lambda_{1,2}=-\beta \pm \gamma$ are the Lyapunov exponents of the system. $\gamma-\beta>0$ implies that the distance of two solutions with neighbouring initial points taken from the same band increases exponentially. Since the unstable manifolds are transversal to the border lines between the bands, we can assume without loss of generality that after $n$ iterations the two solutions arrive at different bands. Once the points are separated in this manner, their orbits can no longer be considered close, since they behave essentially independently.

Since the Lyapunov exponents are known and their sum is always $-2 \beta<0$, the Kaplan-Yorke dimension [Farmer et al., 1983] can be calculated as:

$$
D_{K Y}=1+\frac{\gamma-\beta}{|-\gamma-\beta|} .
$$

$D_{K Y}$ provides an estimate for the fractal dimension of the attractor, thus, we expect to have rather dense attractors at zero damping $(\beta=0)$.

\subsection{Topological Transitivity and Mixing}

To get better insight to the complexity of the examined system, we numerically determined the topological entropy $h_{0}$ at various values of parameter $P$ by the algorithm given in [Chen et al., 1991]. Although certain numerically obtained attractors may seem to stretch over several bands of constant control effort, they can be considered as structures formed by merging of neighbouring repellers. Thus, we measured the topological entropy of such local repellers at several switching lines. The initial regions were chosen to be the parallelograms $Q_{m}$, defined in the previous section, at several values of the index $m$. If the invariant set (or the conditionally invariant set in the case of transient chaos) is larger than $Q_{m}$, the results provide a lower estimate for the topological entropy. According to the algorithm, the pre-images of line sections on the $y$ axis were determined and the number of subsections surviving $1, \ldots, n$ backward iteration steps before leaving the initial domain was detected. The number of backward iterations was allowed to increase from 8 to 24 if the relative error - compared to the previous stage of the calculation - was larger than $0.5 \%$. Each line section was divided into 2500-20000 subsections, depending also on the relative error. The 


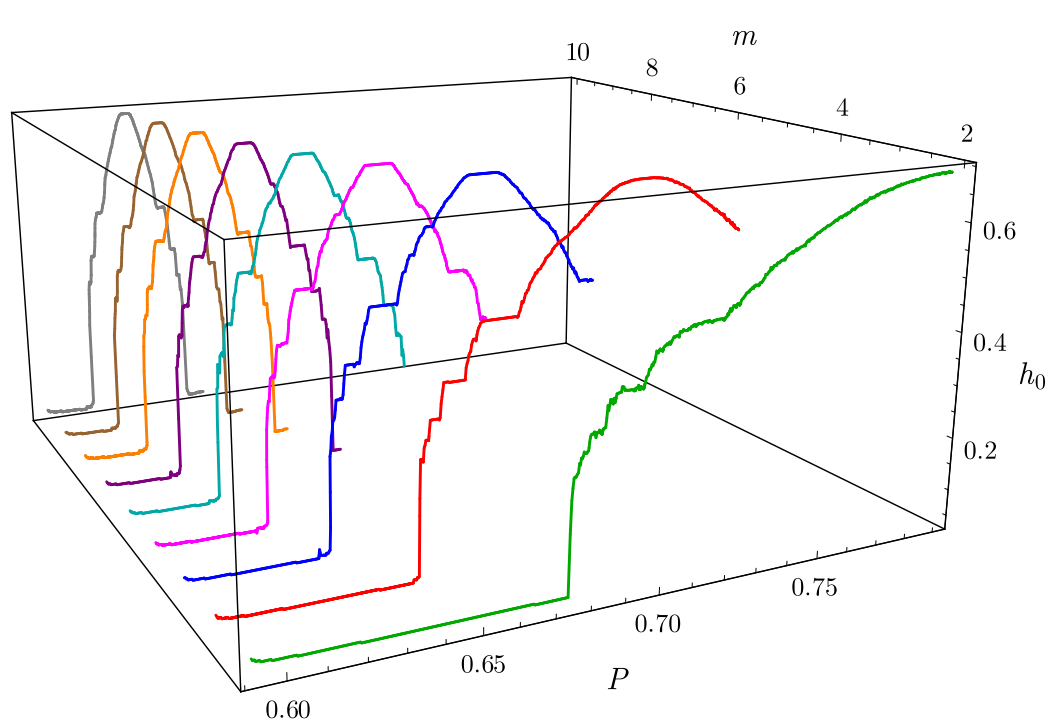

Fig. 6. Topological entropy $\left(h_{0}\right)$ versus parameter $P$ and index of switching line $m$ at $\alpha=0.77, \beta=0.02$ and $D=0.83$.

results are depicted in Fig.6. The devil's staircase structure of the curves is apparent. This property is the consequence of the structural stability of hyperbolic sets. Fig. 7 shows the topological entropy at the switching line $m=3$, with the approximate value of $h_{0}$ at certain levels.

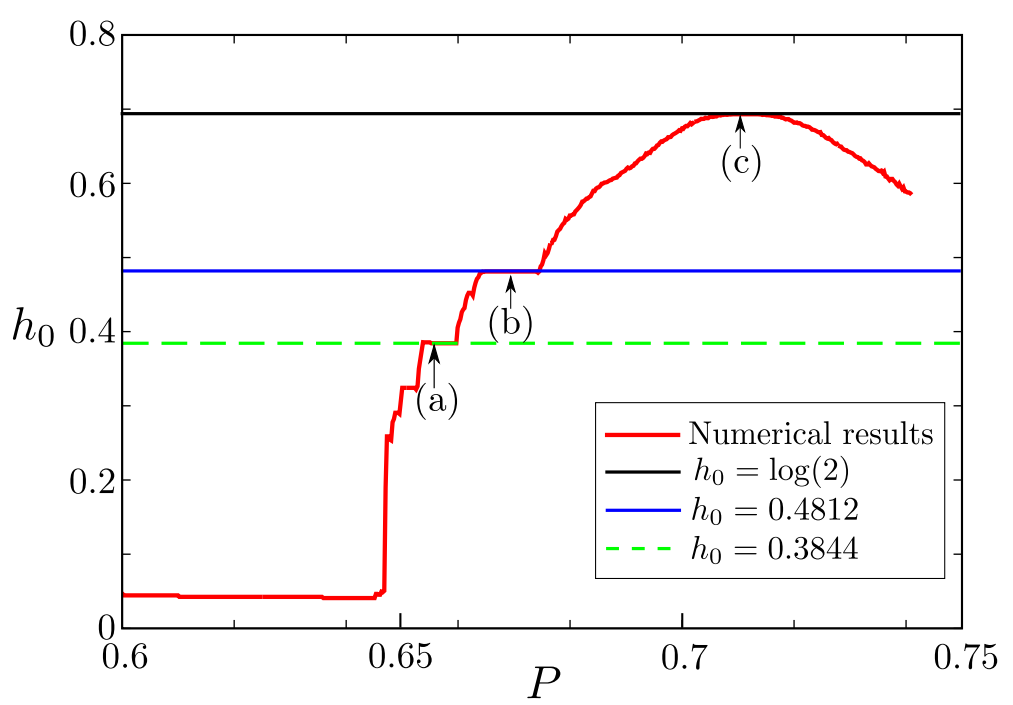

Fig. 7. Topological entropy $\left(h_{0}\right)$ versus parameter $P$ at $m=3, \alpha=0.77, \beta=0.02$ and $D=0.83$.

The images and pre-images of the parallelogram $Q_{m}$ can be calculated analytically. We determined the second pre-images and the third images of the trapezoids on either side of the switching line $S W_{3}$ at the parameters given in the caption of Fig. 7. Parameter $P$ was chosen at three different plateaus of the topological entropy curve. The results are depicted in Fig. 8.

In Fig. 8(c) the images and pre-images fully intersect, forming a Smale horseshoe structure. The numerically obtained topological entropy is very close to $h_{0}=\log (2)$ at this parameter. Indeed, a binary symbolic dynamics can be introduced with symbols denoting the bands on the left and right of the switching line. Thus, the examined dynamical system is conjugate to a full binary shift. This property implies sensitivity on initial conditions, the map is topologically transitive and there exists a countable infinity of periodic orbits, an uncountable infinity of nonperiodic orbits and a dense orbit. Thus, map (13) is chaotic in a finite parameter domain in the neighbourhood of the examined parameters. 

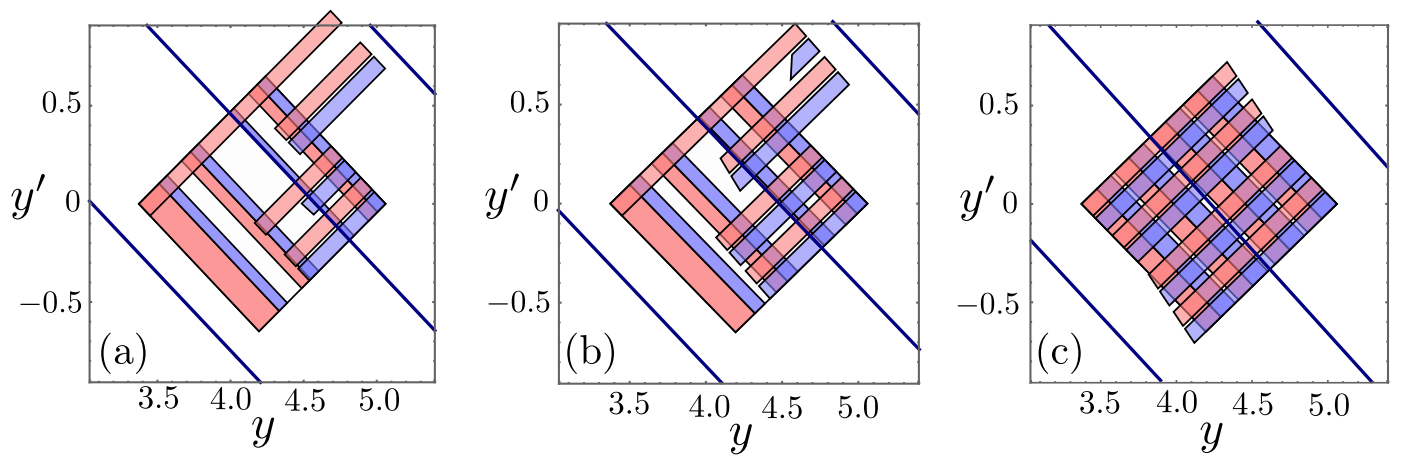

Fig. 8. Second pre-images and third images of the trapezoids left (red) and right (blue) of the switching line $m=3 . \alpha=0.77$, $\beta=0.02$ and $D=0.83$. (a) $P=0.655$, (b) $P=0.67$, (c) $P=0.71$, corresponding to Fig. 7 .

To have a subsystem in the neighbourhood of a certain switching line that is conjugate with a binary shift, the images of the trapezoids $L$ and $R$ (see Fig. 4) must stretch out from the initial parallelogram. That is, $f_{l}\left(P^{L U S W}\right)$ and $f_{l}\left(P^{R U S W}\right)$ must be to the right of the manifold $W_{R}^{S}$, while $f_{r}\left(P^{L U S W}\right)$ and $f_{r}\left(P^{R U S W}\right)$ must be to the left of the manifold $W_{L}^{S}$. The fulfillment of these four escape conditions also ensures that the calculated pre-images are in the appropriate bands, i.e., $\tilde{f}_{l}^{-1}(R) \cap R=\emptyset$ and $\tilde{f}_{r}^{-1}(L) \cap L=\emptyset$ (cf. Eq. (21)). Certainly, one must also ensure that the fixed points exist on both sides of the switching line, i.e., conditions (20) are fulfilled.

The parameter domains where the aforementioned conditions fulfill, are shown in Fig. 9.

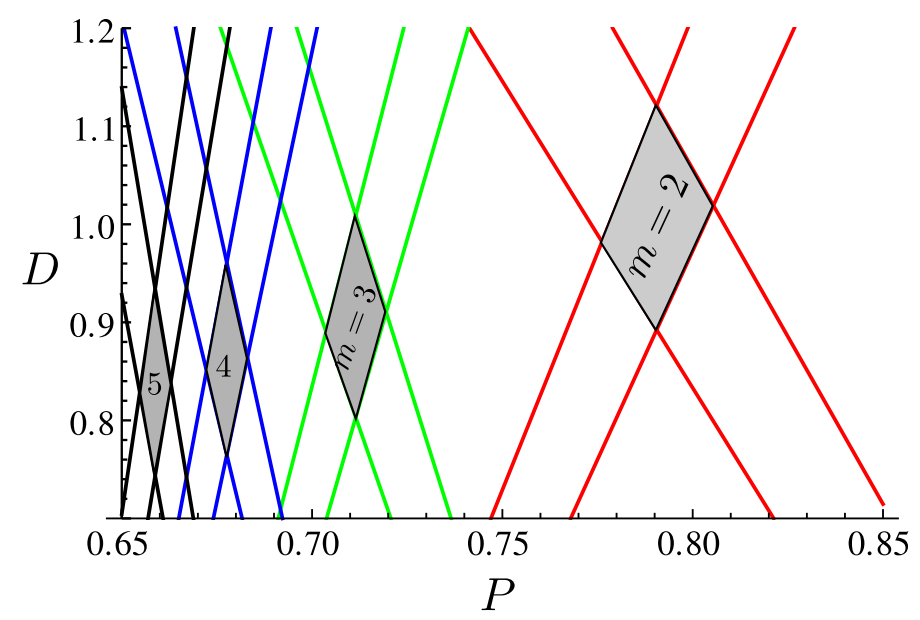

Fig. 9. Parameter domains where the dynamics can be described by a full binary shift at parameters $\alpha=0.77$ and $\beta=0.02$ at the switching lines $m=2,3,4$ and 5 . Lines with the same colour correspond to the four escape conditions evaluated at the same value of $m$.

\section{Conclusions}

Our results show that the small-amplitude stochastic-like vibrations of digitally controlled systems may be related to a deterministic chaotic dynamics descibed by a simple piecewise linear map, the micro-chaos map. The phase-space of the examined PD controlled system contains a finite chain of baker's map structures that can be either open or closed, i.e., solutions may escape from these structures towards the origin or away from the origin. Since the neighbourhood of the origin - the absorbing sphere - is practically stable, the solutions eventually arrive at a chaotic attractor that is either a single baker's attractor or a larger structure that is merged from several repellers. It is worth noting at this point that the size of the absorbing sphere can be important for applications since this value corresponds to the expected error of the control 
system.

In a real control system the sampling times are significantly shorter than the characteristic times of the mechanical system. Consequently, the realistic values of $\alpha, \beta, P$ and $D$ - and the sizes of the attractors - are rather small and the effect of one iteration step of map (13) on the trapezoids $L$ and $R$ is hard to visualize. However, the topology of the phase-space is similar to the one shown in Fig. 5 . The results of a simple cell mapping technique [Hsu, 1987] are depicted in Fig. 10 at parameters of a real experimental device - an inverted pendulum [Enikov \& Stépán, 1998]. The colours in the figure characterize the number of steps necessary to reach an attractor. The triangular red regions in the upper right and lower left corners correspond to points that leave the computational domain in a couple of steps.

The number and size of the individual attractors inside the absorbing sphere vary remarkably as the parameters are changed. The exploration of these phenomena is under progress.

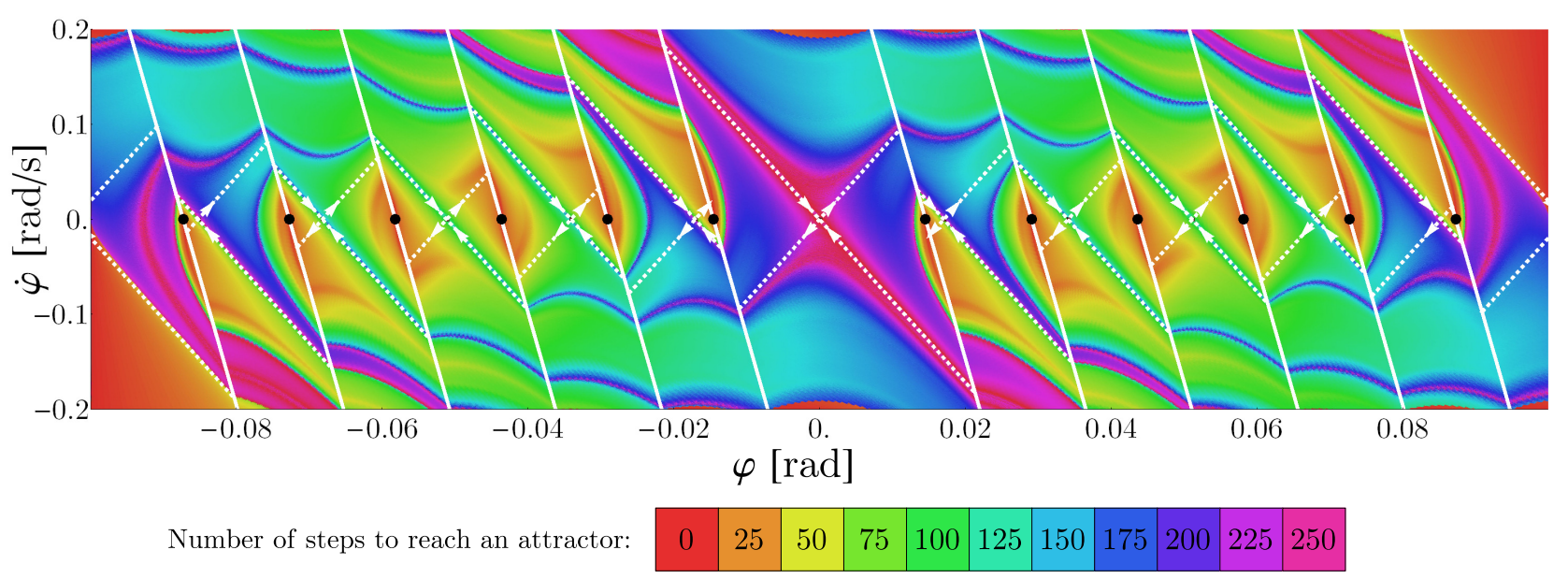

Fig. 10. Cell mapping results showing the domains of attraction (coloured domains) of 12 tiny disconnected attractors (covered by the black points) at realistic parameters $\alpha=6.8511 \cdot 10^{-3}, \beta=0, P=5.4997 \cdot 10^{-5}$, and $D=2.5665 \cdot 10^{-3}$. The switching lines (solid, white) and the stable and unstable manifolds (dashed, white) are also shown. These parameters correspond to an inverted pendulum with mass moment of inertia $M=0.042 \mathrm{~kg} \mathrm{~m}^{2}$ and length $l=0.5 \mathrm{~m}$. The parameters of digital control are $\tau=0.001 \mathrm{~s}$ and $\Delta F=0.024 \mathrm{Nm}$. The maximal control error is about $0.08 \mathrm{rad} \approx 4^{\circ}$.

\section{Acknowledgments}

This research was supported by the Hungarian National Science Foundation under grant no. OTKA K 83890. The research leading to these results has received funding from the European Research Council under the European Union's Seventh Framework Programme (FP/2007-2013) / ERC Advanced Grant Agreement n. 340889.

\section{References}

Cabrera, J.L. \& Milton, J.G. [2004] "Stick balancing: On-off intermittency and survival times", Nonlinear Studies, 11(3) pp. 305-317.

Chen, Q. \& Ott, E. \& Hurd, L.P. [1991] "Calculating topological entropies of chaotic dynamical systems", Physics Letters A 156(1-2) 48-52.

Chen, G \& Dong, X. [1998], From Chaos to Order: Methodologies, Perspectives and Applications, (World Scientific, Singapore).

Csernák, G. \& Stépán, G. [2005] "Life Expectancy of Transient Microchaotic Behaviour" J. Nonlinear Sci. 15(2), 63-91.

Csernák, G. \& Stépán, G. [2007] "Life expectancy calculation of transient chaos in the 2D micro-chaos map", Periodica Polytechnica, 51(2), p. 59-62. 
Csernák, G. \& Stépán, G. [2010] "Digital Control as Source of Chaotic Behavior", International Journal of Bifurcation and Chaos, 20(5), p. 1365-1378.

Csernák, G. \& Stépán, G. [2011] "Sampling and Round-off, as Sources of Chaos in PD-controlled Systems", Proceedings of the 19th Mediterranean Conference on Control and Automation, June 20-23, Corfu.

Csernák, G. \& Stépán, G. [2012] "Disconnected Chaotic Attractors in Digitally Controlled Linear Systems", Recent Researches in Automatic Control, Systems Science and Communications, Proceedings of the 8th WSEAS International Conference on Dynamical Systems and Control, Published by WSEAS Press, ISBN: 978-1-61804-103-6, p. 97-102.

Csernák, G. \& Stépán, G. [2013] "The state-space model of micro-chaos", Proc. the 2013 International Conference on Systems, Control, Signal Processing and Informatics. Rhodes, p. 420-425.

Delchamps, F. D. [1990] "Stabilizing a linear system with quantized state feedback", IEEE Trans. Autom. Contr., 35 p. 916-924.

Enikov, E. \& Stépán, G. [1998] "Micro-Chaotic Motion of Digitally Controlled Machines" J. of Vibration and Control 4, p. 427-443.

Farmer, J. D. \& Ott, E. \& Yorke, J. A. [1983] "The dimension of chaotic attractors", Physica, 7D p. 153-180.

Garay, B. M. \& Csikja, R. \& Tóth, J. [2008] "Some chaotic properties of the beta-hysteresis transformation", Proc. of the 2008 International Symposium on Nonlinear Theory and its Applications, Budapest, Hungary, September 7-10, pp. 191-194.

Haller, G. \& Stépán, G. [1996] "Micro-Chaos in Digital Control", J. Nonlinear Sci., 6, p. 415-448.

Hsu, C.S. [1987] Cell-to-Cell Mapping. A Method of Global Analysis for Nonlinear Systems, Applied Mathematical Sciences, Vol. 64. (Springer-Verlag, New York), ISBN: 978-0-387-96520-8

Kuo, B. C. [1977] Digital Control Systems, (SRL Publishing, Champaign, IL, USA), ISBN-13: 9780195120646.

Lakshmikantham, V. \& Leela, S. \& Martynyuk, A. A. [1990] Practical Stability of Nonlinear Systems, (World Scientific, Singapore), ISBN: 978-981-02-0351-1.

Milton, J.G. \& Bélair, J. [1990] "Chaos, Noise and Extinction in Models of Population Growth", Theoretical Population Biology, 37(2).

Trefethen, L. N. \& Embree, M. [2005] Spectra And Pseudospectra: The Behavior of Nonnormal Matrices And Operators (Princeton University Press, USA), ISBN: 9780691119465.

Widrow, B. \& Kollár, I. [2008] Quantization Noise: Roundoff Error in Digital Computation, Signal Processing, Control, and Communications, (Cambridge University Press, Cambridge, UK), 778 p., ISBN-13: 9780521886710, ISBN: 0521886716. 